\title{
Enhancing the Radio Link Protocol for VoIP Session Establishment Signalling over Satellite-UMTS
}

\author{
Victor Y.H. Kueh, Rahim Tafazolli, Barry Evans \\ Mobile Communications Research Group \\ Centre for Communication Systems Research (CCSR), University of Surrey \\ Guildford, Surrey, United Kingdom GU2 7XH \\ V.Kueh@eim.surrey.ac.uk
}

\begin{abstract}
Session Initiation Protocol (SIP) is an application layer signalling protocol used in the IP-based UMTS network for establishing multimedia sessions. With a satellite component identified to play an integral role in UMTS, there is a need to support SIP-based session establishment over Satellite-UMTS (SUMTS) as well. Due to the inherent characteristics of SIP, the transport of SIP over an unreliable wireless link with a larger propagation delay is inefficient. To improve the session setup performance, a link layer retransmission based on the Radio Link Control acknowledgement mode (RLC-AM) mechanisms is utilised. However the current UMTS RLC-AM procedure is found to cause undesirable redundant retransmission when applied over the satellite. As such, this paper proposes an enhancement to the RLC protocol through a timer-based retransmission scheme. Simulation results reveal that not only the system capacity can be improved through this redundant retransmission avoidance scheme, but also better system performances in terms of session setup delay and failure are gained.
\end{abstract}

\section{INTRODUCTION}

Due to the enormous success and popularity of the Internet, IP is rapidly emerging as the lingua franca of all future networking with the next generation of mobile communication systems set to evolve to become IP-based as well. The emergence of IP-based network for mobile communications will enable the convergence of, and the access to voice, video, messaging, data and web-based technologies for the wireless user in a seamless manner, in much the same way the fixed users are currently experiencing over the Internet. The provision of these IP-based multimedia services is made possible through the introduction of the IP Multimedia Subsystem (IMS) as part of the 3GPP Release 5 set of standards in Universal Mobile Telecommunication Systems (UMTS). Session Initiation Protocol (SIP) [1], a protocol developed within the Internet Engineering Task Force (IETF), has been selected by the 3GPP as the end-to-end IP signalling protocol for establishing multimedia sessions.

At the same time, a satellite component has been identified in UMTS, where in contrast to the $2^{\text {nd }}$ generation mobile global satellite systems which were conceived as stand-alone systems, each with its own proprietary protocol, the third generation offers the unique opportunity of a fully integrated and standardized satellite segment with open interfaces including the air interface. In addition to its fast service deployment and coverage extension capability, satelliteUMTS (S-UMTS), as a direct consequence of its broadcast nature and ubiquitous coverage, offers a natural way to provide multicast and broadcast services in a more costefficient manner than its terrestrial counterpart. To achieve high degree of commonality with the IP-based T-UMTS network, there is therefore a need to support SIP-based session establishment over the satellite component as well. Integration and co-operation of S-UMTS with the terrestrial system is necessary as this will not only enable a seamless service provision, but it also permits the re-use of the terrestrial infrastructure as well as the use of highly integrated multimode terrestrial/satellite terminals. All these result in reduced technology complexity and overall system cost.

Nevertheless, the transport of SIP over the radio interface is not efficient due to the inherent characteristics of SIP being transactional-based and generous in size. This is made worse when transversing an error-prone wireless link with a larger satellite propagation delay. One of the techniques to reduce the session setup delay is by compression of the signalling messages, such as the text-based message compression technique proposed in [2]. Our approach to improve the session setup performance is by incorporating a link layer retransmission based on the Radio Link Control acknowledgement mode (RLC-AM) [3] mechanisms as a method to conceal link-related losses from the upper layers.

In [4], we have shown that by adding unsolicited STATUS report trigger on top of solicited feedback, the session setup delay and blocking probability are reduced due to the increased responsiveness of the RLC error recovery process. However, this configuration of having more than one trigger for the transmission of the STATUS report is found to cause unnecessary retransmissions when applied over the satellite. This redundancy is undesirable since it lowers the protocol efficiency. In view of this, an enhancement to the RLC protocol based on a timer retransmission scheme is proposed in this paper to overcome this inefficiency.

The paper is organized as follows. In Section II, the inefficiency of the current UMTS RLC-AM procedure when applied over the satellite is highlighted, of which a scheme proposed to alleviate this will be presented. Section III describes the simulation environment and assumptions, and 
the simulation results are reported in Section IV. Finally, conclusions are drawn in Section V.

\section{ENHANCEMENT TO RLC-AM FOR SATELLITE-UMTS}

The RLC-AM retransmission method as defined in UMTS is based on a hybrid sliding window ARQ protocol with selective acknowledgments (SACK) and negative acknowledgments (NAK). Higher layer data packets, known as RLC Service Data Units (SDUs) are segmented into fixed length RLC Packet Data Units (PDUs). Feedback in the form of a STATUS report is sent to indicate to the sender which PDUs have been successfully received and which have not. Based on the STATUS report received, the sender retransmits those negatively acknowledged PDUs. With many features provided, RLC-AM is extremely flexible and can be configured in several ways.

However a problem has been identified with the use of the current UMTS RLC-AM procedure over the satellite environment. It has been found that when more than one STATUS report trigger is configured for transactional applications such as session establishment, as in the case of having both the unsolicited and solicited STATUS report trigger options set, redundant retransmission is more likely to occur. This happens when the sender retransmits a PDU more than once in response to the received STATUS report although earlier retransmission has already taken place. This is because due to the long round trip time over the satellite, the first retransmission of the PDU may still be in-flight and thus has not reached the receiver. As a result, the next generated STATUS report is not up-to-date and the already retransmitted PDU is still indicated as missing. An example of this is explicitly illustrated in Fig. 1.

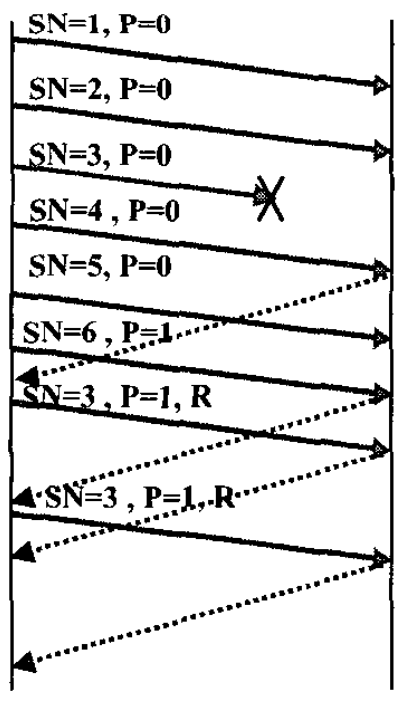

\section{AMD PDU}

\section{Status PDU}

Fig. 1. Example of the occurrence of redundant retransmission due to outdated feedback
In the example shown, the PDU with $\mathrm{SN}=3$ is missing. When the subsequent PDU, i.e. the PDU with $\mathrm{SN}=4$, reaches the receiver successfully, a STATUS report is generated to report back to the sender that the $\mathrm{PDU}$ with $\mathrm{SN}=3$ is missing, since the 'Missing PDU Indicator' has been configured [3]. The sender then retransmits the PDU with $\mathrm{SN}=3$ upon receiving the unsolicited STATUS report. While this retransmitted PDU is still in-flight, another STATUS report is triggered, where this time it is transmitter-driven due to poll bit set in the PDU with $\mathrm{SN}=6$ (the last $\mathrm{PDU}$ of the SDU). Since the $\mathrm{PDU}$ with $\mathrm{SN}=3$ has yet arrived at the receiver (due to the high satellite round trip time), this PDU is again reported as missing. Another retransmission of this PDU will ultimately cause redundant retransmission when both of the retransmissions of the same PDU are successfully received later.

This redundant retransmission is undesirable as it is rendered useless and ignored by the receiver. Since there is no true information transfer, extra retransmission only leads to a waste in the channel bandwidth. Secondly, since retransmissions of PDUs have a higher priority than new PDUs, this will only block the transfer of new PDUs. Furthermore, redundant retransmission consumes battery power needlessly which is not energy efficient and with mobile terminal powered by a finite battery source, it is therefore pivotal to conserve this limited energy resource. Moreover, these extra retransmissions unnecessarily increase the interference to other users and in an interference-limited system, as in a CDMA environment, this is detrimental as it may globally deteriorate the quality of service.

Hence a scheme, which we named 'RLC-AM Timer-based Retransmission', is proposed. This approach associates each PDU with the time when it was last (re)transmitted, i.e. every time a PDU is transmitted or retransmitted, the time at that moment is taken. Every time retransmission of a PDU is triggered, be it due to the reception of a STATUS report (solicited or unsolicited) or the expiry of the poll timer (when there is no new PDU for transmission), a comparison is made between the time of the last (re)transmission of that PDU with the current time. If the difference is more than the round trip time of the RLC PDU, another retransmission of this PDU is allowed to take place, otherwise the retransmission is suppressed. Thus it can be seen that the implementation of this scheme requires a variable associated with each PDU, which saves the time it is last (re)transmitted. Since this scheme requires only minor additions locally (without affecting the operation of its peer entity if this scheme is not supported therein), it is fully backward compatible with the existing implementation of UMTS RLC-AM.

\section{SimUlation MOdEl AND ASSUMPTIONS}

To compare the performance of the proposed scheme, RLC-AM Timer-based Retransmission with the standard RLC procedure as specified in [3], herein referred to as UMTS RLC-AM (Baseline), a system level simulator developed in OPNET $^{1}$ similar to that described in [4] was used. These

' OPNET is a trademark of Opnet Technologies inc 
schemes were assessed for an end-to-end mobile originated SIP-based voice over IP (VoIP) session establishment to a fixed user over the Internet through the UMTS Satellite Radio Access Network (USRAN), the UMTS IP Core Network and the IMS. The reference architecture employed, which is consistent with the UMTS Release 5 IP-based network architecture [5], is shown in Fig. 2. The session establishment signalling sequences followed the ones depicted in [4] and were modelled according to their protocol behaviour as detailed in their respective specifications with the message sizes following the ones listed in [6]. It was assumed that the user equipment (UE) was located within the service area of the network operator to whom the UE subscribed and that the UE had already setup an RRC connection and activated a primary PDP context which was used to carry the IMS related signalling. It was also assumed that the UE had performed registration with both the GPRS network as well as with the IMS network.

The USRAN consists of a satellite and an S-UMTS gateway, whereby in the selected scenario, the satellite is considered to be a transparent Geostationary Earth Orbit (GEO) satellite, while the Node B and Radio Network Controller (RNC) are collocated in the gateway. The roundtrip delay over the UMTS IP Core Network was assumed to be $150 \mathrm{~ms}$ [7], while the mean one-way Internet delay and its standard deviation are $50 \mathrm{~ms}$ and $7 \mathrm{~ms}$, respectively [8]. The processing time per SIP message in each server is $25 \mathrm{~ms}$ [2] while the RLC processing time was assumed to be $15 \mathrm{~ms}$ for both uplink and downlink communications [9]. It was assumed that there is no loss in the fixed network, while for the air interface, an erroneous link with parameterized block error rate (BLER) was considered, whereby the errors were assumed to be uniformly distributed (uncorrelated). For the RLC setting, segmentation and in-sequence delivery options were enabled, and only the 'last PDU in transmission buffer', 'last PDU in retransmission buffer' and 'timer based' polling triggers were considered in the simulation. The 'SDU discard after MaxDAT number of transmissions' was chosen for the SDU discard function, while for the STATUS report, both the unsolicited and solicited STATUS report transfers were enabled. The rest of the parameters used in the simulations are summarized in Table I. Note that the values used for these parameters are either those recommended or typical values as listed in the respective specifications.

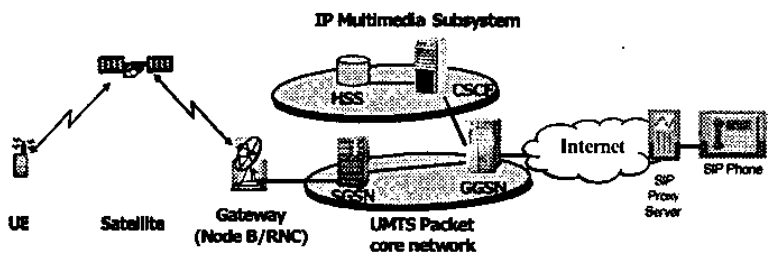

Fig. 2. IP-based S-UMTS reference network architecture
TABle 1. Simulation Parameter SetTings

\begin{tabular}{|l|c|l|c|}
\hline SIP Timer, T1 & $2 \mathrm{~s}$ & Timer T3380 & $30 \mathrm{~s}$ \\
\hline SIP Timer, T2 & $16 \mathrm{~s}$ & Timer T3381 & $8 \mathrm{~s}$ \\
\hline SIP Timer, T4 & $17 \mathrm{~s}$ & RLC Window Size & 1024 \\
\hline RSVP R Vajue & $30 \mathrm{~s}$ & RLC Poll Timer & $0.8 \mathrm{~s}$ \\
\hline RSVP K Value & 3 & $\begin{array}{l}\text { RLC Poll Prohibit } \\
\text { Timer }\end{array}$ & $1.6 \mathrm{~s}$ \\
\hline $\begin{array}{l}\text { Max. Tx. of } \\
\text { Activate Sec. PDP } \\
\begin{array}{l}\text { Context Request } \\
\text { \& Modify PDP } \\
\text { Context Request }\end{array}\end{array}$ & 5 & RLC MaxDAT & 4 \\
\cline { 3 - 4 } & & TT1 & $10 \mathrm{~ms}$ \\
\cline { 2 - 4 } & & Data Rate & $32 \mathrm{kbps}$ \\
\hline
\end{tabular}

The performance metrics used in this comparison study are as follows:

- Session setup delay

The session setup delay is defined to be the interval between entering the last dialled digit and receiving a ringback.

\section{- $\quad$ Call blocking probability}

The probability that the session setup fails after the maximum attempt of transmission of any of the higher layer messages is reached.

\section{- $\quad$ SDU discard rate}

The SDU discard rate is defined to be the percentage of SDUs discarded out of the total number of SDUs transmitted.

\section{- Extra retransmission}

This redundancy indicator is the ratio of the number of transmissions of an RLC Acknowledgement Mode Data (AMD) PDU ignored by the receiver to the total number of RLC AMD PDUs transmitted.

\section{SimULATION RESULTS AND DISCUSSION}

Fig. 3 compares the extra retransmissions performance of the proposed scheme with the standard RLC-AM scheme for different BLERs. As expected, the proposed scheme exhibits a lower number of redundant retransmission than the UMTS RLC-AM (Baseline) procedure, of which the difference between the two increases with the BLER. These redundant retransmissions due to the receipt of out-of-date STATUS report can cause the PDU to reach its MaxDAT value unnecessarily. As a result, the UMTS RLC-AM (Baseline) procedure has a much higher SDU discard rate compared to the RLC-AM Timer-based Retransmission scheme, as illustrated in Fig. 4. The higher the SDU discard rate, the more the RLC needs to rely on retransmissions from the upper layers for error recovery. With more retransmissions triggered at the upper layers, the session establishment will eventually fail when the maximum attempt of any of the upper layer protocols is reached. As a result, it can be seen from Fig. 5 that the UMTS RLC-AM (Baseline) procedure has a higher call blocking probability than the proposed method. 


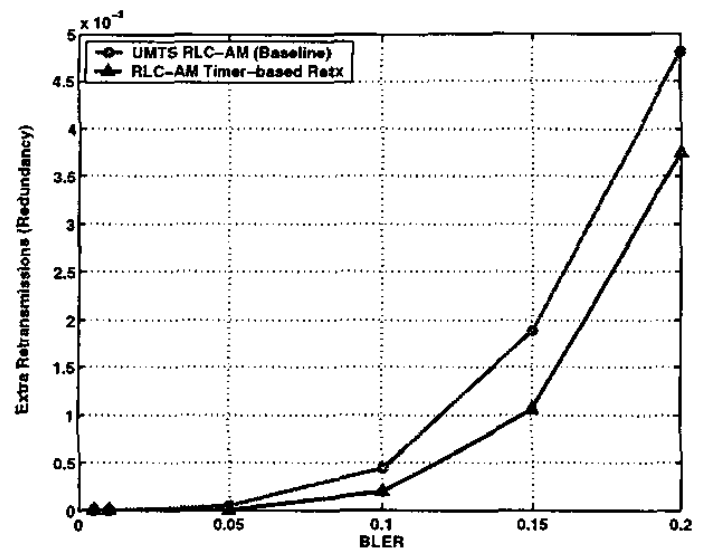

Fig.3. Extra retransmission comparison between timer-based Retx with UMTS RLC scheme for different BLERs

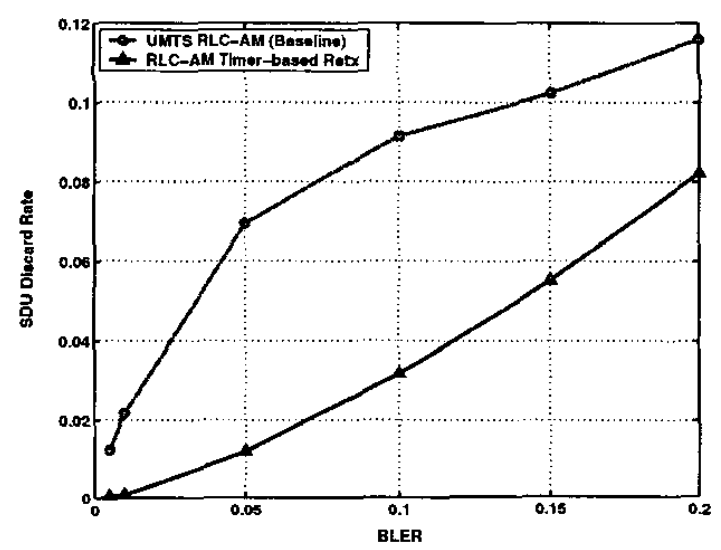

Fig.4. SDU discard rate comparison between timer-based Retx with UMTS RLC scheme for different BLERs

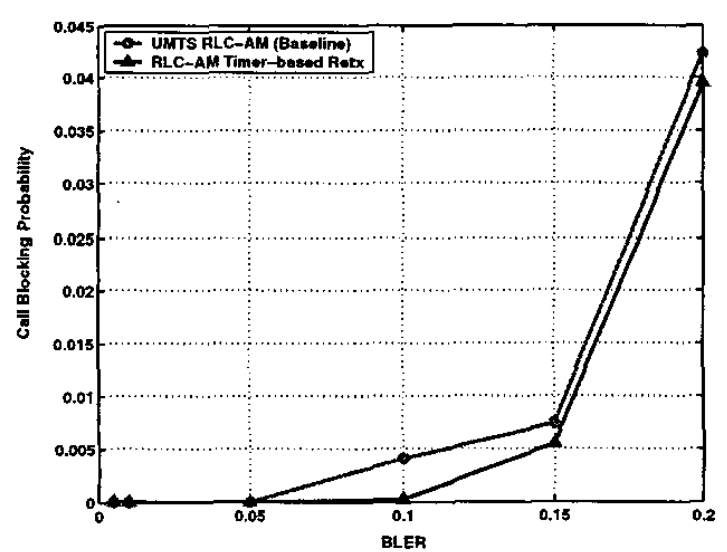

Fig.5. Call blocking probability comparison between timer-based Retx with UMTS RLC scheme for different BLER

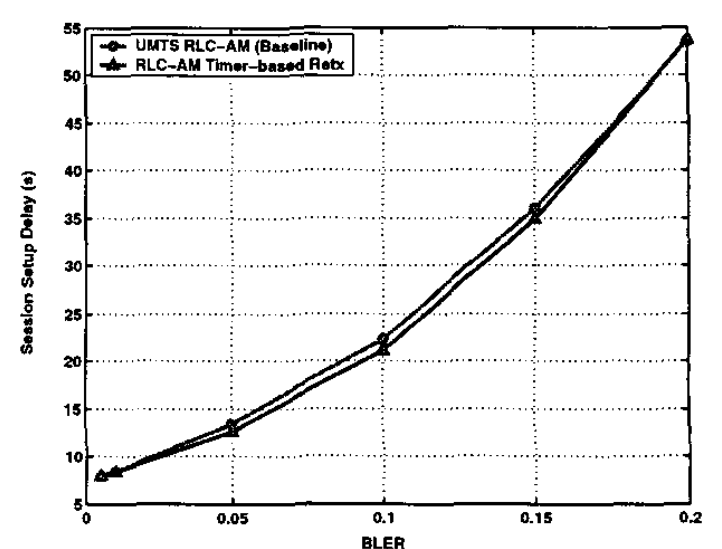

Fig.6. Session setup delay comparison between timer-based Retx with UMTS RLC scheme for different BLERs

Fig. 6 shows that an improved session establishment delay is obtained with the timer-based retransmission scheme. Due to the higher SDU discard rate, the UMTS RLC-AM (Baseline) scheme needs to rely on the upper layers to ensure reliability, whereby the end-to-end error recovery process is generally slower compared to local retransmissions performed at the link layer. It is noted that the improvement of the RLCAM Timer-based Retransmission scheme over the UMTS RLC-AM (Baseline) is more obvious at moderate BLER. This is because at low BLER, there are not so many AMD or control PDUs that are lost, while at high BLER, both schemes suffer from high PDU lost. Hence the advantage of the proposed scheme at these two extreme values of BLER is not as apparent.

\section{CONCLUSIONS}

In this paper, we have proposed a timer-based retransmission scheme, which allows a PDU retransmission to take place only when the difference between the time of the last (re)transmission of that PDU with the current time is more than the RLC PDU round trip time. This scheme is able to reduce the number of redundant retransmissions which occur due to the unnecessary triggering of retransmission when the received STATUS report is not up-to-date. This condition happens when more than one STATUS report trigger is enabled, as in the case when both the unsolicited and solicited feedbacks are configured, as well as when the round trip time of the RLC PDU is high, as in transversing a GEO satellite. With a lower SDU discard rate attained with this scheme, the session establishment failure and delay performances are eventually improved. Therefore, this scheme not only increases the protocol efficiency but also the system capacity can be improved as lower interference is acquired through this duplicate retransmission avoidance scheme.

\section{REFERENCES}

[1] J. Rosenberg, H. Schulzrinne, G. Camarillo, A. Johnston, J. Peterson, R. Sparks, M. Handley and E. Schooler, "SIP: Session Initiation Protocol," Request for Comments 3261, IETF, June 2002. 
[2] G. Foster, M. Pous, A. Sesmun, V. Kenneally and D. Pesch, "Performance Evaluation of UMTS Packet Voice Call Control," 5th European Personal Mobile Communications Conference (EPMCC 2003), Glasgow, Scotland, April 2003, pp. 136-140.

[3] 3GPP TS 25.322 V5.3.0: "Radio Access Networks; RLC Protocol Specification; protocol specification (Release 5)," December 2002.

[4] V. Y. H. Kueh, R. Tafazolli, B. Evans, "Performance Evaluation of SIP. based Session Establishment over Sateilite-UMTS," IEEE Vehicular Technology Conference (VTC) Spring 2003, Jeju, Korea, 22-25 ${ }^{\text {th }}$ April 2003.

[5] 3GPP TR 23.821 V.1.0.1: "Architecture Principles for Release 2000, (Release 2000)," July 2000.

[6] GP-000508, 3GPP TSG GERAN, "A Comparison Between PacketSwitched Call Setup Using SIP and GSM Circuit-Switched Call Setup Using RIL3-CC, RIL3-MM, RIL3-RR, and DTAP," Nortel Networks, November 2000.

[7] H. Holma and A. Toskala, "WCDMA for UMTS," John Willey \& Sons, 2002.

[8] T. J. Kostas, M. S. Borella, I. Sidhu, G. M. Schuster, J. Grabiec, and J. Mahler, "Real-time voice over packet-switched networks," IEEE Network, no. 1, January/Febnuary 1998, pp. 18-27.

[9] 3GPP TR 25.853 V.4.0.0: "Delay Budget within the Access Stratum (Release 4)," March 2001. 